\title{
Role of PET/CT in post-therapeutic assessment of bronchogenic carcinoma
}

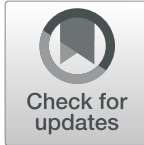

\author{
Amira Ayman Shaheen ${ }^{1,2^{*}}$ (D), Ahmed Mostafa Mohammed ${ }^{1}$, Ahmed Elshimy ${ }^{1}(\mathbb{D}$ ) and \\ Mennatallah Hatem Shalaby ${ }^{1}$ (i)
}

\begin{abstract}
Background: Lung cancer is the most common among all kinds of cancers. It still constitutes the leading cause of cancer-related deaths worldwide, even with major advancements in prevention and treatments available. More than $85 \%$ of the cases are of non-small cell lung cancer (NSCLC), while less than 15\% are of small cell lung cancers (SCLCS).

Patients and methods: This is a prospective study of 20 patients confirmed histopathologically to have bronchogenic carcinoma, who came for assessment of therapeutic response. All patients underwent positron emission tomography/computed tomography (PET/CT) before and after therapy. Semiquantitative assessment was used to determine maximum standardized uptake value (SUVmax). Treatment response evaluation was assessed according to the Response Evaluation Criteria in Solid Tumors (RECIST) criteria.
\end{abstract}

Results: Comparison of the pre- and post-treatment SUVmax in the responder and non-responder groups revealed that the post-treatment SUV was significantly lower than the baseline SUV in the responder group $(P=0.008)$. The responder post-treatment SUV and $\triangle$ SUV were significantly lower than the non-responder values $(P=0.014$ and 0.0004 respectively). The optimum threshold values of post-treatment SUV and $\triangle$ SUV threshold defined by the receiver operating characteristic (ROC) curve analysis were $\leq 8$ and $\leq-48.3$ respectively. The sensitivity, specificity, PPV, NPV, and AUC of post-treatment SUV for predicting tumor response were 100\%,66.67\%, 66.7\%, 100\%, and 0.833 respectively. The sensitivity, specificity, PPV, NPV, and AUC of $\triangle$ SUV for predicting tumor response were $100 \%$, $91.67 \%, 88.9 \%, 100 \%$, and $0.979 \%$ respectively.

Conclusion: PET/CT proved itself as useful, efficient, and reliable tool in follow-up of lung cancer patients as it gives an early and accurate metabolic response assessment before any $\mathrm{CT}$ changes, leading to early modification of therapy or confirmation of its efficiency.

Keywords: Bronchogenic carcinoma, Treatment response, PET/CT, SUVmax, RECIST

\section{Background}

The goal of therapy of the NSCLC patients is mainly dependent on the stage of the disease as follows: patients with stage I to III disease, the goal is to cure, whereas for those patients with stage $I V$, the goal is to relieve the symptoms and to continue life. The goal of therapy in

\footnotetext{
* Correspondence: amira_shaheen@hotmail.com;

AmiraShaheen@med.asu.edu.eg

'Radiology Department, Faculty of Medicine, Ain Shams University, Cairo, Egypt

${ }^{2}$ Cairo, Egypt
}

patients with SCLC is to cure, which can be obtained through combined therapy modality with chemotherapy and radiation [1].

There is a need for a multidisciplinary methodology to include the advanced imaging techniques for the purpose of early accurate staging of lung cancer and administrating the treatment in order to avoid ineffective treatments to improve the chances of overall survival. This in turn will be reflected on the patient's quality of life [2].

\section{Springer Open}

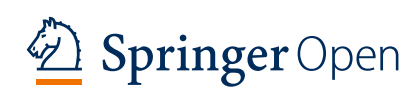

(c) The Author(s). 2021 Open Access This article is licensed under a Creative Commons Attribution 4.0 International License, which permits use, sharing, adaptation, distribution and reproduction in any medium or format, as long as you give appropriate credit to the original author(s) and the source, provide a link to the Creative Commons licence, and indicate if changes were made. The images or other third party material in this article are included in the article's Creative Commons licence, unless indicated otherwise in a credit line to the material. If material is not included in the article's Creative Commons licence and your intended use is not permitted by statutory regulation or exceeds the permitted use, you will need to obtain permission directly from the copyright holder. To view a copy of this licence, visit http://creativecommons.org/licenses/by/4.0/. 
Until now, therapy-induced change in tumor size measured by CT according to the RECIST [3-6] criteria has been considered as a surrogate marker of identification of therapeutic efficacy. Recently, PET/CT provides other tumor criteria including, density and texture changes, alteration of vascularity on computed tomography (CT), intensity of 18 fluorodeoxyglucose (FDG) uptake on positron emission tomography (PET)$\mathrm{CT}$ on PET-CT were considered as promising biomarkers for a faster and more accurate assessment of response to therapy [7].

Because of the strong positive relationship between tracer uptake and the number of viable cancer cells, PET response criteria in solid tumors (PERCIST) were proposed in 2009 not to replace RECIST criteria but to complement them. It depends on standardized uptake value (SUV uptake) changes by the tumoral mass and has a unique advantage of predicting response by assessing the change in tumor metabolism [8].

Combination of both CT and FDG PET together improves the early diagnosis and accuracy level of lung cancer, as the changes in the cellular metabolism occur more rapidly than causing changes in the tumor size [9]. By obtaining information of the metabolic activity of the tumor cells, FDG PET has become a useful and powerful tool in initial staging and follow-up of lung cancer patients [2].

Fluorodeoxyglucose (FDG) is known to be the most common radiotracer used in PET imaging. Studies have shown that the 18F-FDG uptake by tumor cells, as assessed by SUVmax, is a significant prognostic factor in the treatment response of lung cancer. SUVmax has been widely used as an indicator of tumor metabolic activity because of its rapid speed [10].

Standardized uptake value (SUV) is calculated by normalizing the attenuation-corrected FDG uptake of the injected dose and body weight in the lesion. SUVmax is a voxel with the maximum uptake of FDGs in the region of interest (ROI) [11, 12].

Tumor progression through first-line chemotherapy occurs in approximately one third of patients with lung cancer. This high rate of progression underlines the need to track treatment response with advanced imaging modalities, which can potentially help in treatment decision $[2,13]$.

Using CT in monitoring the rapidly growing tumors with central necrosis may not be beneficial in size change detection. Therefore, using PET/CT metabolic parameters that integrate both uptake intensity and tumoral volume may be very helpful in follow-up of the necrotic tumors, post-therapeutic changes, or tumors associated with distal atelectasis $[2,13]$.

Radiation-induced lung injury is one of the challenges of the post-treatment assessment of lung cancer. It generally manifests in two phases: an early phase of transient radiation pneumonitis, which typically occurs within the first 6 months, and a later phase of chronic radiation fibrosis, which usually occurs at 6-12 months after completion of radiotherapy [2, 10, 14].

Thus, performing PET/CT study at least 3 months after completion of radiotherapy is suggested to decrease the risk of false-positive results [14]. However, persistent FDG uptake associated with radiation-induced fibrotic or inflammatory changes occasionally lasts for 15 months after the end of therapy. This highlights the significant role of PET/CT in differentiation of the tumoral mass from the nearby pulmonary changes $[2,14]$.

The aim of this study is to elucidate the role of FDG $\mathrm{PET} / \mathrm{CT}$ in assessment of treatment response in patients with bronchogenic carcinoma.

\section{Methods}

\section{Patients}

This is a prospective study conducted at our institute during the period from September 2018 to September 2020. A total of 20 patients (18 males and 2 females) with a histopathological diagnosis of bronchogenic carcinoma were included in this study. Their ages ranged from 33 to 79 years with a median of 58 years. They were provided with a signed informed consent, and the study was conducted after institutional review board approval by Radiology Department Scientific Board as well as fulfilling the ethical guidelines of the institute. Patients with a known diagnosis and pathologically proven bronchogenic carcinoma were referred by clinical oncologists and pulmonologists for follow-up after chemotherapy and/or radiotherapy to assess response of treatment.

\section{Inclusion criteria}

All patients with a known diagnosis and pathologically proven bronchogenic carcinoma were included in this study with no age or sex predilection. Only the primary tumor was assessed whether there is nodal or distal metastasis or not.

\section{Exclusion criteria}

Patients excluded were those who had blood glucose level more than $200 \mathrm{mg} / \mathrm{dl}$, underwent surgery, or did not receive chemo/radiotherapy.

\section{Patient preparation}

Total fasting for $6 \mathrm{~h}$ was requested before the study. Diabetic patients were asked to avoid high carbohydrate diet and allowed high protein diet and liquids. Blood glucose level at the first and second PET/CT scans in diabetic patients was in the range of $110-160 \mathrm{mg} / \mathrm{dl}$ at the time of the study. Pre-procedural assessment of patient's 
weight, height, and serum creatinine was done before the study and confirmed to be within normal limits for CT contrast injection $(0.7-1.4 \mathrm{mg} / \mathrm{dL})$. Administration of ante-cubital intravenous cannula and injection of $18 \mathrm{~F}$ FDG were done. Patients were instructed to stay quiet in the injection room for average $60 \mathrm{~min}$ and were asked to drink plain water and to avoid any kind of strenuous activity including chewing prior the procedure. An average of 5-10 mCi of 18FDG was administered for each patient 50-60 min before examination. After the procedure, patients were advised to drink water as much as they could to washout any remaining radioactive material, and to avoid contact with pregnant women or children for at least 6-8 $\mathrm{h}$ after the examination.

\section{Technique}

All patients underwent FDG PET/CT examination using Discovery IQ GE machine. The patients were introduced to the PET-CT machine, lying in supine position with head fixation and arms up for image acquisition, starting from the skull base down to the upper thigh level. Lowdose non-enhanced CT scan was first performed for attenuation correction, then a whole-body PET study followed by diagnostic enhanced whole-body CT scan. The whole study lasted for approximately $20 \mathrm{~min}$. Hundreds of trans-axial PET and CT images were firstly reconstructed and then reformatted into coronal and sagittal images to facilitate image interpretation. For each of these sets of PET and CT images, corresponding "fusion" images, combining the two types of data, were also generated.

\section{Image interpretation}

Interpretation of PET/CT images was done by two specialized radiologists with at least 5 years' experience. Decisions were reached by consensus.

Staging of primary tumors of each case was done according to 8th edition TNM classification [15]. Pretreatment and post-treatment follow-up 18F-FDG-PET/ CT were performed with an interval of 3-5 months (median $=4$ months) between the two scans. We used RECI ST 1.1 criteria on CT interpretation as a gold standard. In CT images, bronchogenic carcinoma assessment was based on size while in PET images semiquantitative measurements of metabolic uptake in FDG-avid primary tumor lesion in pretreatment and post-treatment scans were performed and then compared and evaluated for their potential to predict treatment response. SUVmax was the parameter used for semiquantitative assessment and potential differentiation of lesions. Patients with an outcome of a complete response (CR) or a partial response (PR) were subsequently classified as responders according to RECIST criteria. However, those who had an outcome of stable disease (SD) or progressive disease (PD) were defined as non-responders.

\section{Statistical analysis}

Statistical analysis was performed using the MedCalc statistical software for Windows (MedCalc Software, Mariakerke, Belgium). Data for continuous variables were expressed as either median, interquartile range and range or mean \pm standard deviation, and as both number and percentage for categorical data. Mann-Whitney $U$-test was used to evaluate the differences in continuous variables between responder and non-responder groups. The Wilcoxon test for paired samples was used to compare the pre- and post-treatment parameters in responder and non-responder groups while the $T$-test for paired samples was used to compare the pre- and posttreatment parameters in $\mathrm{PD}, \mathrm{SD}$, and $\mathrm{PR}$, and group comparisons of all parameters between the PD, SD, PR, and CR groups were performed using the Kruskal-Wallis test, and Conover post hoc test was used for pairwise comparisons of the different groups. Receiver operator characteristic (ROC) curve analysis was performed to determine optimum thresholds and the diagnostic accuracy of the various variables in distinguishing the different groups. The diagnostic accuracy of all variables was evaluated in terms of sensitivity, specificity, positive predictive value (PPV), negative predictive value (NPV), and area under the ROC curve (AUC). Chi-squared test was used for comparison of categorical data between the two groups. Pearson's correlation was used to describe the relationship between SUV and size. For all tests, all $P$ values were two-tailed, and a $P$-value $<0.05$ was considered significant.

\section{Results}

Characteristics of the included patients $(N=20)$ with bronchogenic carcinoma are presented in Table 1.

Comparison of the pre- and post-treatment SUV in the responder and non-responder groups revealed that the post-treatment SUV was significantly lower than the baseline SUV in the responder group while no significant difference existed between post-treatment and baseline values in the non-responder group (Table 2, Figs. 1 and 2).

When responder and non-responder groups were compared, no significant difference was found between the pre-treatment SUV of both groups; however, the responder post-treatment SUV and $\Delta$ SUV were significantly lower than the non-responder values (Table 3).

Since post-treatment SUV and $\Delta$ SUV differed significantly between responder and non-responder groups, receiver operating characteristic (ROC) curve analysis was performed to determine the optimal threshold values of 
Table 1 Demographic parameters and clinical data of the patients

\begin{tabular}{ll}
\hline Parameter & Number of cases \% \\
\hline Age $(y r)$ & $56.4 \pm 12.6$ years \\
Sex & $18(90 \%)$ \\
Male & $2(10 \%)$ \\
Female & \\
Smoking & $13(65 \%)$ \\
Yes & $7(35 \%)$ \\
No & \\
Stage & $7(35 \%)$ \\
II & $5(25 \%)$ \\
III & $8(40 \%)$ \\
IV & \\
Pathological type & $13(65 \%)$ \\
Adenocarcinoma & $2(10 \%)$ \\
Squamous cell carcinoma & $5(25 \%)$ \\
SCLC & \\
RECIST & $1(0.5 \%)$ \\
Complete response & $7(35 \%)$ \\
Partial response & $9(45 \%)$ \\
Stable disease & $3(15 \%)$ \\
Progressive disease &
\end{tabular}

these parameters for differentiation of responders from non-responders (Table 4).

When the area under the ROC curves for the posttreatment SUV and $\triangle$ SUV were compared to determine which variable was better in distinguishing responders and non-responders, no significant difference was found between the area under the curve (AUC) of both curves $(P=0.128)$; however, the AUC for $\triangle \mathrm{SUV}$ was greater than that of the post-treatment SUV (Fig. 3).

In an effort to determine whether SUV can differentiate between the individual groups, namely, the PD, SD, PR groups, comparisons of the various variables between the 3 groups were also performed. The CR group was composed of a single patient and was therefore excluded from the formal statistical analysis of the individual groups due to the inadequate sample size. No significant difference was seen between baseline and post-treatment SUV in the SD and PD groups; however, the posttreatment SUV was significantly lower than the pretreatment SUV in the PR group (Table 5).

When PD, SD, and PR groups were compared, no significant difference was found between the pre- and posttreatment SUV of all groups; however, there was a significant difference among the $\triangle \mathrm{SUV}$ of all groups. $\triangle \mathrm{SUV}$ of the PD group was significantly higher than both SD and PR groups, and the $\triangle \mathrm{SUV}$ of the SD group was significantly higher than the PR group (Table 6, Figs. 4 and 5).

Since $\triangle S U V$ differed significantly between PD, SD, and PR groups, ROC curve analysis was performed to determine the optimum threshold value for $\triangle S U V$ for differentiation of PD, SD, and PR groups. A $\triangle \mathrm{SUV}$ of $>15.4$ could distinguish PD group from the SD group with a sensitivity, specificity, PPV, and NPV of 100\%, 100\%, $100 \%$, and $100 \%$ respectively. PR could be distinguished from SD using a threshold value for $\triangle \mathrm{SUV}$ of $\leq-48.3$ with a sensitivity, specificity, PPV, and NPV of 100\%, $88.89 \%, 87.5 \%$, and $100 \%$ respectively. $\triangle$ SUV values ranging from $>-48.3$ to $\leq 15.4$ designated SD with a sensitivity, specificity, PPV, and NPV of $88.89 \%, 100 \%, 100 \%$, and $87.5 \%$ respectively. CR by definition means total disappearance of the lesion with a $\triangle \mathrm{SUV}=-100$, so even though CR was excluded from the statistical analysis, any lesion with a SUV $=-100$ would indicate $C R$ and $\triangle$ SUV values from $>-100$ to $\leq-48.3$ designated PR with a sensitivity, specificity, PPV, and NPV of $100 \%, 88.89 \%$, $87.5 \%$, and $100 \%$. The slight overlap between the $\Delta$ SUV values of the PR and SD groups is due to single adenocarcinoma patient with stable disease course but a $\triangle \mathrm{SUV}$ value as low as -54 .

Since the metabolic activity of a mass is likely to be influenced by its size, the possible correlation between the SUV and the size of the lesion was also assessed. No significant correlation was found between baseline SUV values and baseline size values $(r=0.33, P=0.16)$; however, a moderate positive correlation was found between post-treatment SUV values and post-treatment sizes. As the size increased so did the SUV $(r=0.53, P=0.02)$. The strongest correlation was demonstrated between $\triangle$ SUV and $\Delta$ size $(r=0.91, P<0.0001)$ whereas $\Delta$ SUV values increased from negative to positive indicating an

Table 2 Comparison of baseline and post-treatment SUV in both responder and non-responder groups

\begin{tabular}{|c|c|c|c|c|c|c|}
\hline & \multicolumn{3}{|c|}{ Responder group $(n=8)$} & \multicolumn{3}{|c|}{ Non-responder group $(n=12)$} \\
\hline & Pre-treatment & Post-treatment & $P$-value & Pre-treatment & Post-treatment & $P$-value \\
\hline \multicolumn{7}{|l|}{ SUV } \\
\hline Median & 14.6 & 5.6 & & 10.9 & 11.8 & \\
\hline IQR & $11.8-17.7$ & $2.8-6.3$ & 0.008 & $4.95-20.7$ & $6.3-19.4$ & 0.638 \\
\hline Range & $10-20$ & $0-8$ & & $3.5-34.7$ & $2-28.2$ & \\
\hline
\end{tabular}




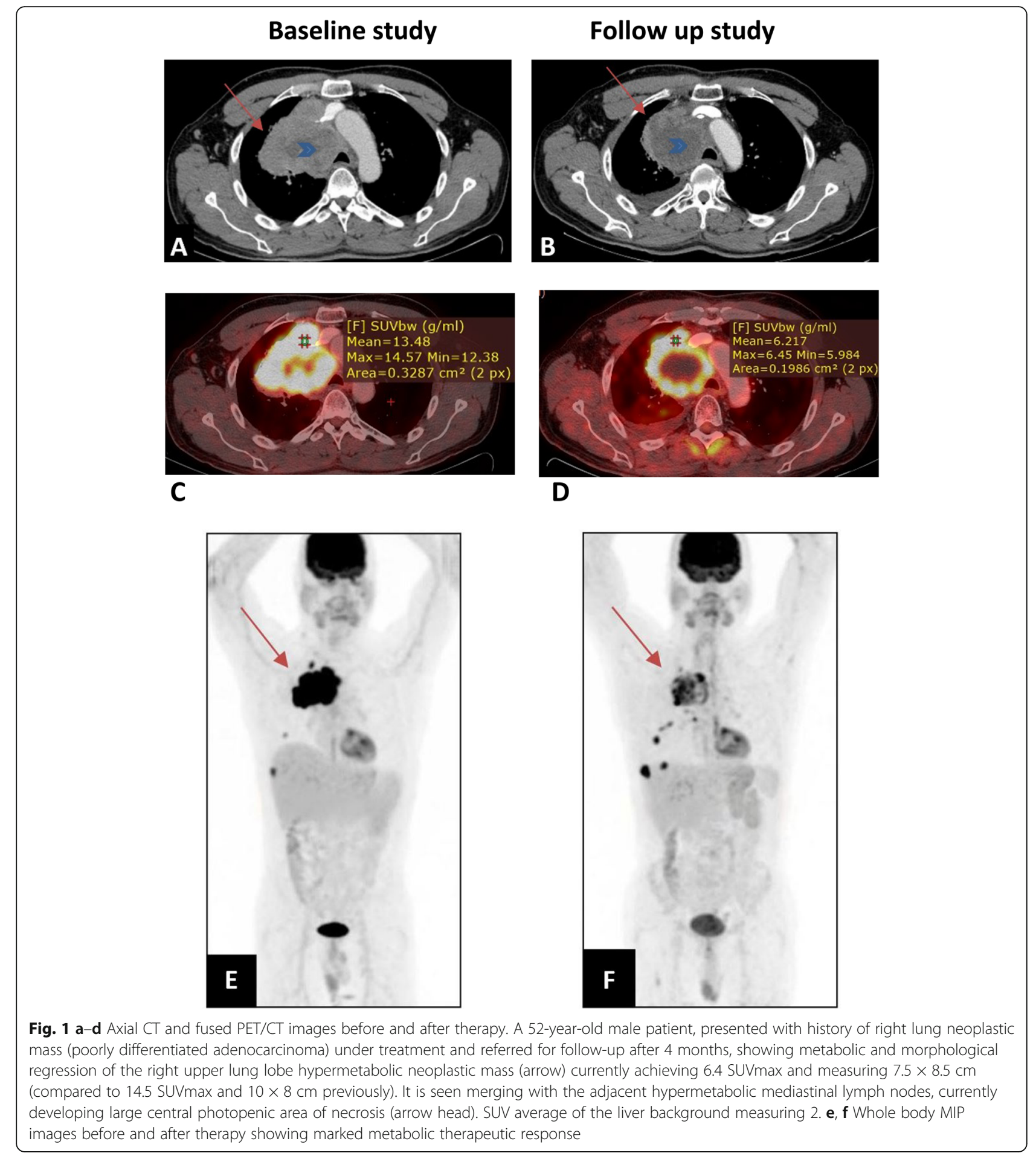

increase in post-treatment SUV as compared to baseline SUV; the $\Delta$ size of the lesion also increased from negative to positive indicating an increase in the posttreatment size as compared to baseline size. This is to be expected as metabolic progression is almost always accompanied by an increase in the size of the lesion (Fig. 6).

\section{Discussion}

The aim of the current study was to elucidate the role of $\mathrm{PET} / \mathrm{CT}$ in assessment of treatment response of patients with bronchogenic carcinoma.

Several studies highlighted the effectiveness of 18FFDG-PET/CT in the post-treatment assessment and follow-up of lung cancer patients. 


\section{Baseline study}
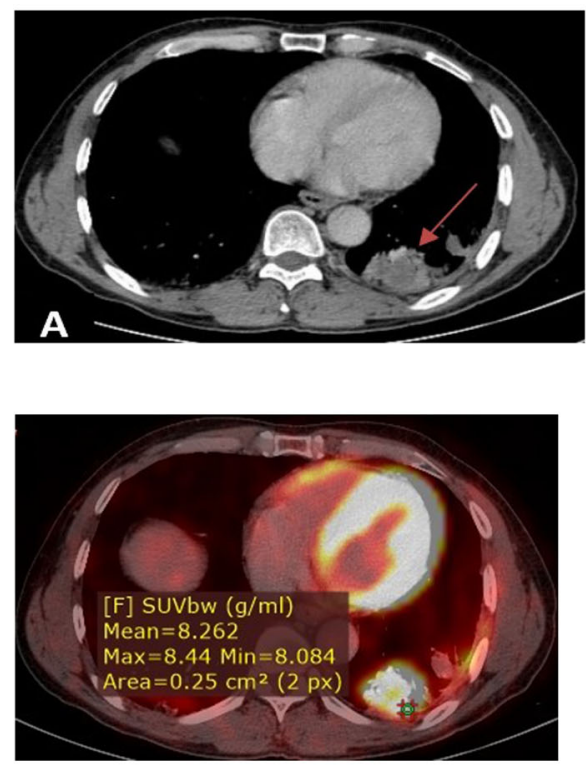

C

\section{Follow up study}
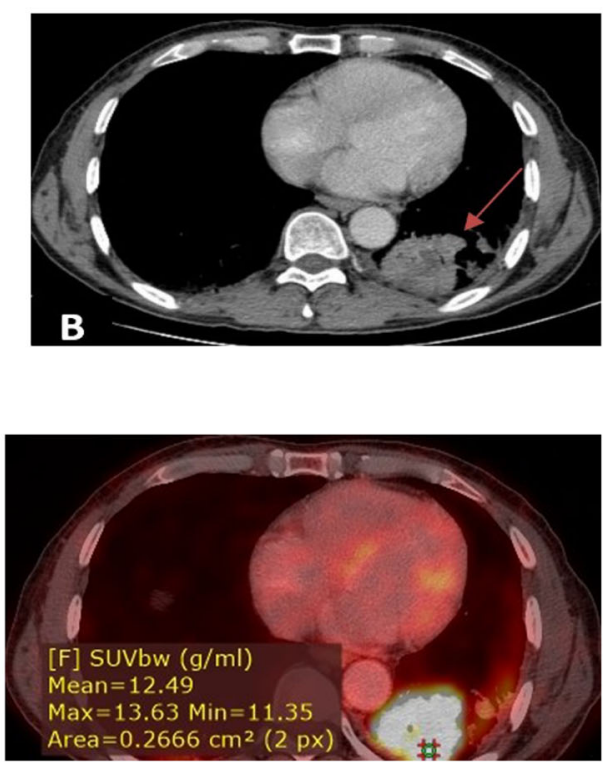

D
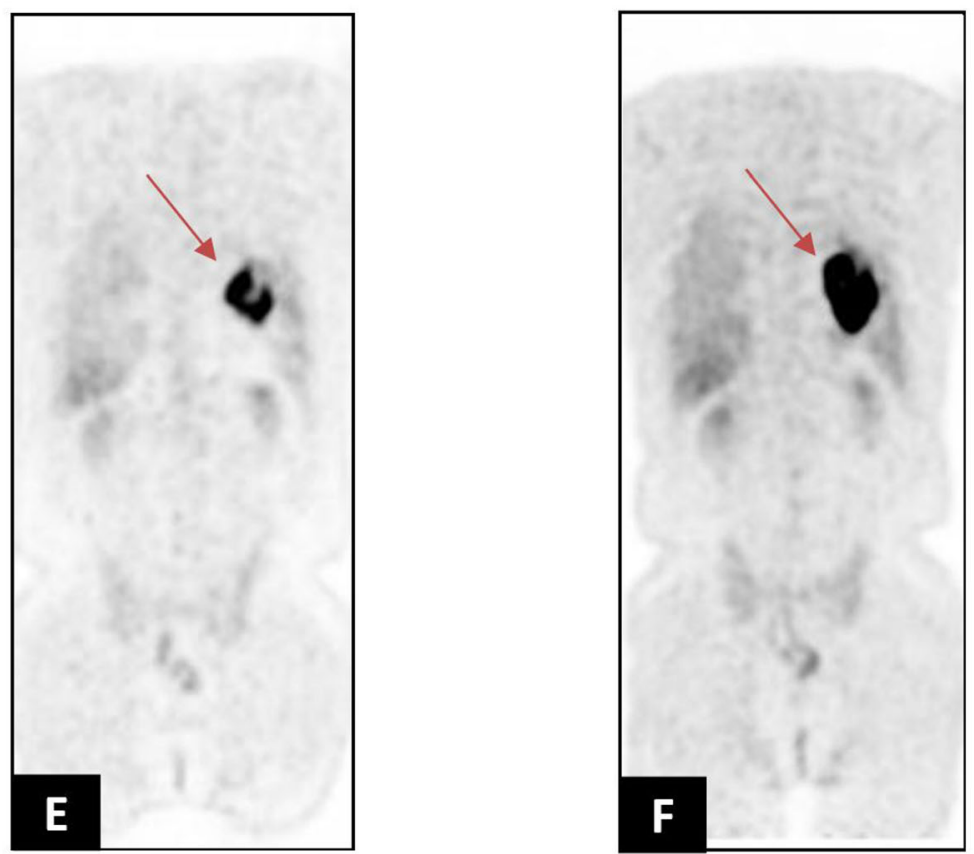

Fig. 2 a-d Axial CT and fused PET/CT images before and after therapy. A 59-year-old male patient, with history of left-sided bronchogenic cancer (adenocarcinoma), received chemotherapy and referred for follow-up after 3 months, showing interval progression in the size and metabolic activity of the previously reported hypermetabolic left lung lower lobar soft tissue mass lesion (arrow) measuring about $4.5 \times 5.5 \mathrm{~cm}$ and achieving 13.6 SUVmax on current examination (compared to $3.1 \times 3.4 \mathrm{~cm}$ and achieving 8.4 SUVmax on previous study). SUV average of the liver background measuring 1.5. e, f Whole body MIP images before and after therapy showing progression of the primary left lung neoplasia

This study enrolled 20 patients $(18$ males and 2 females). Their ages ranged from 33 to 79 years with a median of 58 years, an IQR of $50-66.5$ years, and a mean $( \pm$ standard deviation) of 56.4 years ( \pm 12.6 years). The number of males was significantly higher than the number of females in this study. In the current study, histopathological analysis of the lung tumors revealed the presence of 13 (65\%) adenocarcinomas, 2 (10\%) squamous cell carcinomas, and $5(25 \%)$ small cell lung cancers. Sixteen $(80 \%)$ and $4(20 \%)$ tumors were located in the right and 
Table 3 Comparison between pre-treatment SUV, posttreatment SUV, and \% change in SUV ( $\triangle S U V$ ) of the responder and non-responder groups

\begin{tabular}{llll}
\hline & Responder group & Non-responder & $P$-value \\
\hline \multicolumn{2}{c}{ Pre-treatment SUV } & & \\
Median & 14.6 & 10.9 & \\
IQR & $11.8-17.7$ & $4.95-20.7$ & 0.396 \\
Range & $10-20$ & $3.5-34.7$ & \\
Post-treatment SUV & & \\
Median & 5.6 & 11.8 & 0.014 \\
IQR & $2.8-6.3$ & $6.3-19.4$ & \\
Range & $0-8$ & $2-28.2$ & \\
$\begin{array}{lll}\Delta S U V \\
\text { Median }\end{array}$ & -63.1 & & 0.0004 \\
IQR & -83.1 to -53.4 & -32.8 to 54.9 & \\
Range & -100 to -48.3 & -54 to 113.5 & \\
\hline
\end{tabular}

left lungs respectively. No significant difference was seen between the number of smokers $(13,67.5 \%)$ and nonsmokers $(7,35 \%)$ in this study $\left(x^{2}=1.8, P=0.18\right)$; however, a significant association between smoking and gender was noted $\left(x^{2}=3.92, P=0.05\right)$ where a significantly larger number of smokers in this study were males.

In agreement with this study, Akl et al. included 404 confirmed cases of bronchogenic carcinoma to assess the clinicopathological profile of the bronchogenic carcinoma cases. They reported that male to female ratio was 4.6:1. The highest incidence was in the sixth and seventh decades of life (63.6\%). Smoking was found to be the main risk factor in $75.7 \%$ of patients. Four types of bronchogenic carcinoma were found: squamous cell carcinoma $37.4 \%$, adenocarcinoma $29.5 \%$, small cell carcinoma $14.9 \%$, large cell carcinoma $7.2 \%$, and undifferentiated carcinoma $11.1 \%$ [16].

Regarding TNM staging, in this study, we used the new 8th TNM classification staging system in controversy to most of the studies which used the older 7th edition of TNM staging as in a study done by Chao and Zhang [17].

Many studies have reported the effect of PET/CT in the accurate $\mathrm{T}$ staging and tumor delineation from surrounding pulmonary reactions, and this is consistent with Chao and Zhang, Steinert, Aydin et al., and Hochhegger et al. who concluded that PET/CT measurements

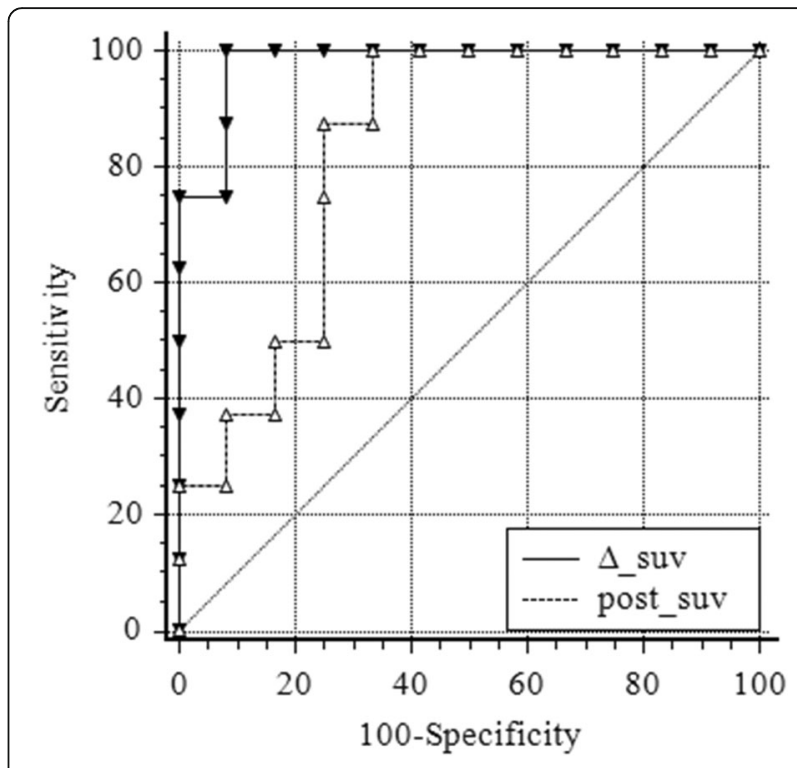

Fig. 3 Receiver operating characteristic curve analyses. The specificity and sensitivity of using post-treatment SUV and \% change in SUV were compared. The AUC was 0.979 (95\% Cl, 0.930-1.000) and $0.833(95 \% \mathrm{Cl}, 0.651-1.000)$ for $\%$ change in SUV and posttreatment SUV respectively. However, no significant difference in AUCs was identified between \%change in SUV and post-treatment $\operatorname{SUV}(P=0.1284)$. AUC, area under the curve; $\mathrm{Cl}$, confidence interval

are more accurate and compatible with histopathological size when compared to $\mathrm{CT}$, and this will affect $\mathrm{T}$ staging of the tumor [17-20].

In this study, we found out that comparison of the pre- and post-treatment SUV in the responder and nonresponder groups revealed that the post-treatment SUV was significantly lower than the baseline SUV in the responder group $(P=0.008)$ while no significant difference existed between post-treatment and baseline values in the non-responder group, and this is almost close to the results found by Bahce et al. and Yamamoto et al. who studied the ability of post-therapeutic tumor SUV uptake to predict pathological response in patients with NSCLC and compared the results with the histopathology. They found a significant difference in the SUVmax on preand post-treatment FDG PET scans of patients who responded to treatment versus the scans of patients who did not have a response [21, 22].

In this study, we reported that when responder and non-responder groups were compared, no significant

Table 4 The sensitivities, specificities, positive predictive values (PPV), negative predictive values (NPV), area under the curves (AUC), and $P$-values of the optimum threshold values of the post-treatment SUV and SUV for differentiation of responders from nonresponders

\begin{tabular}{llllllll}
\hline Parameter & Optimum threshold value & Sensitivity & Specificity & PPV & NPV & AUC & $P$-value \\
\hline Post-treatment SUV & $\leq 8$ & $100 \%$ & $66.67 \%$ & $66.67 \%$ & $100 \%$ & 0.833 & 0.0003 \\
$\triangle$ SUV & $\leq-48.3$ & $100 \%$ & $91.67 \%$ & $88.9 \%$ & $100 \%$ & 0.979 & $<0.0001$ \\
\hline
\end{tabular}


Table 5 Comparison of baseline and post-treatment SUV in PD, SD, and PR groups

\begin{tabular}{|c|c|c|c|c|c|c|c|c|c|}
\hline & \multicolumn{3}{|c|}{ Progressive disease $(N=3)$} & \multicolumn{3}{|c|}{ Stable disease $(N=9)$} & \multicolumn{3}{|c|}{ Partial response $(N=7)$} \\
\hline & Pre-treatment & Post-treatment & $P$-value & Pre-treatment & Post-treatment & $P$-value & Pre-treatment & Post-treatment & $P$-value \\
\hline \multicolumn{10}{|l|}{ SUV } \\
\hline Mean & 5.97 & 12.37 & 0.14 & 17.23 & 13.41 & 0.09 & 14.04 & 5.3 & 0.0006 \\
\hline SD & 3.85 & 8.54 & & 11.25 & 8.82 & & 3.15 & 2.17 & \\
\hline
\end{tabular}

$N$ number, $S D$ standard deviation

difference was found between the pre-treatment SUV of both groups $(P=0.396)$; however, the responder posttreatment SUV and $\triangle$ SUV were significantly lower than the non-responder values. This was in concordance with Huang et al. who investigated the role of SUVmax to predict the short-term outcome of chemo-radiotherapy in patients with advanced NSCLC and concluded that changes in SUVmax values were significantly lower in responders than in non-responders according to RECIST criteria which was used as a gold standard. The sensitivity, specificity, and accuracy of SUVmax change for predicting tumor response were $83.3,84.6$, and $84.9 \%$ respectively [23].

This agrees with Cerfolio et al. who studied the change of SUV to assess the effectiveness of chemo-radiotherapy in patients with NSCLC based on histopathological analysis, and reported that reduction in the $\triangle$ SUVmax of more than $80 \%$ can significantly predict a complete pathological response with a sensitivity of $90 \%$, specificity of $100 \%$, and accuracy of $96 \%$. Also, Lee et al. concluded that single PET/CT scan taken after one cycle of treatment could predict pathological response and can be used as a measure to avoid ineffective treatment. They stated that a reduction of SUVmax by more than $20 \%$ was used as a criterion for a partial metabolic response (PMR) and an increase of SUVmax by more than $25 \%$ as criterion for progressive metabolic disease (PMD). Metabolic response was compared with conventional radiographic response according to WHO criteria $[24,25]$.
In addition to a study by Eschmann et al. who evaluated FDG-PET for assessment of therapeutic response and for prediction of patient outcome after radiochemotherapy of patients with NSCLC, PET findings were compared with the histology of tumor samples. That study demonstrated that the sensitivity, specificity, and accuracy of SUVmax were 95, 80, and 91\%, respectively [26].

In this study, assessment of therapy response using CT established by using RECIST 1.1 criteria, as radiologic assessment of size change and reduction of tumoral mass, thus proves as an extremely useful surrogate marker for quick assessment of tumor response to treatment and is now one of the methods of assessment of choice in most clinical trials to assess drug efficacy [27]. In this study, we revealed that the strongest correlation was demonstrated between $\triangle$ SUV and $\Delta$ size $(r=0.91, P<0.0001)$. This agrees with Fattah et al., Cappabianca et al., Lu et al., and Khalaf et al. who reported that the tumor size and the entity of necrosis are some of the factors that affect the SUVmax of a tumor with positive correlation between tumor diameter and SUV$\max [28-31]$.

This study is almost close to the result found by Weber et al. who studied the use of FDG-PET/CT to predict response to chemotherapy in patients with advanced NSCLC and found close correlation between

Table 6 Comparison between pre-treatment SUV, post-treatment SUV, and \% change in SUV ( $\triangle S U V$ ) of the PD, SD, and PR groups

\begin{tabular}{llll}
\hline & Progressive disease & Stable disease & Partial response \\
\hline Pre-treatment SUV & & & \\
Median & 4 & 14.3 & 13.9 \\
IQR & $3.63-8.8$ & $9.43-25.78$ & $11.7-16.05$ \\
Range & $3.5-10.4$ & $3.5-34.7$ & $10-19.19$ \\
Post-treatment SUV & & & \\
Median & 8.1 & 15.4 & 6 \\
IQR & $7.13-18.68$ & $5.6-18.18$ & $4.56-6.38$ \\
Range & $6.8-22.0$ & $2-28.2$ & $1.1-8.0$ \\
$\Delta$ SUV & & & -09 \\
Median & 102.5 & -18.7 & -57.5 \\
IQR & $96.35-110.75$ & -44.23 to 7.43 & -75.0 to -51.7 \\
Range & $94.3-113.5$ & -54 to 15.4 & -88.9 to -48.3 \\
\hline
\end{tabular}




\section{Baseline study}
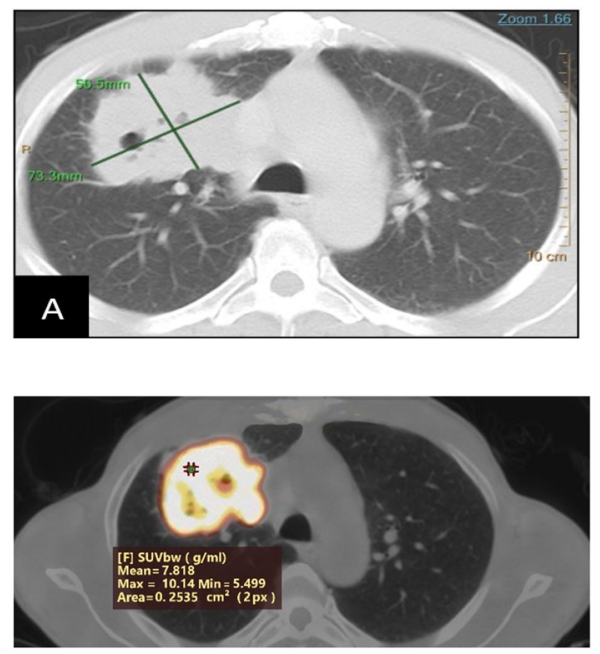

C
Follow up study
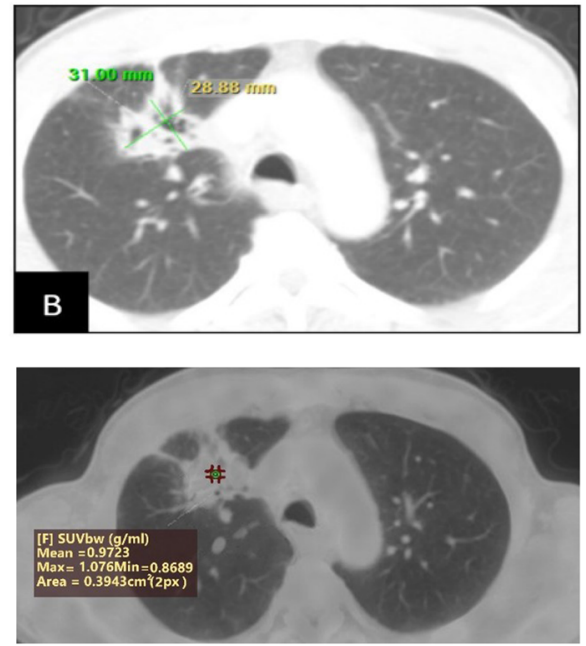

D

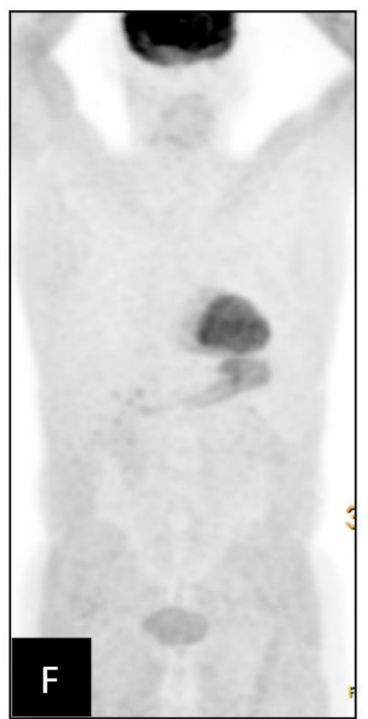

Fig. 4 a-d Axial CT and fused PET/CT images before and after therapy. A 55-year-old male patient with history of right lung cancer received chemo and radiotherapy. Referred for follow-up after 3 months, showing complete resolution of the neoplastic right upper lobe cavitary soft tissue mass activity (currently not exceeding $1.1 \mathrm{SUV}$ max), compared to $10 \mathrm{SUVmax}$ previously. It also shows remarkable regression measuring now $3.3 \times 3 \mathrm{~cm}$ (compared to $7.3 \times 5.6 \mathrm{~cm}$ previously). SUV average of the liver background measuring 1.7. e, $\mathbf{f}$ Whole body MIP images before and after therapy showing the complete therapeutic response of the right upper lobe neoplastic mass

metabolic response and best response to therapy according to RECIST criteria $(P<.0001$; sensitivity and specificity for prediction of best response, $95 \%$ and $74 \%$, respectively) [32].

In controversy, Sheikhbahaei et al. reported that although CT is the modality of choice on posttherapeutic assessment of lung cancer using RECIST criteria which is widely accepted and used for assessment, yet in case of irregular and spiculated lesions, it shows variable results due to variable observer views in size measurement. In addition, activity changes which occur after treatment cannot be detected by CT. Another limitation is the inability of CT to differentiate viable tumor accurately from posttherapeutic inflammation and fibrosis, or even benign changes such as consolidation [2].

It is also concordant with the study by Hicks et al. who reported that using $\mathrm{CT}$ in monitoring the rapidly growing tumors undergoing central necrosis, size change might not be detected, while PET/CT metabolic parameters that integrate both uptake intensity and tumoral volume are very helpful in follow-up of the necrotic 


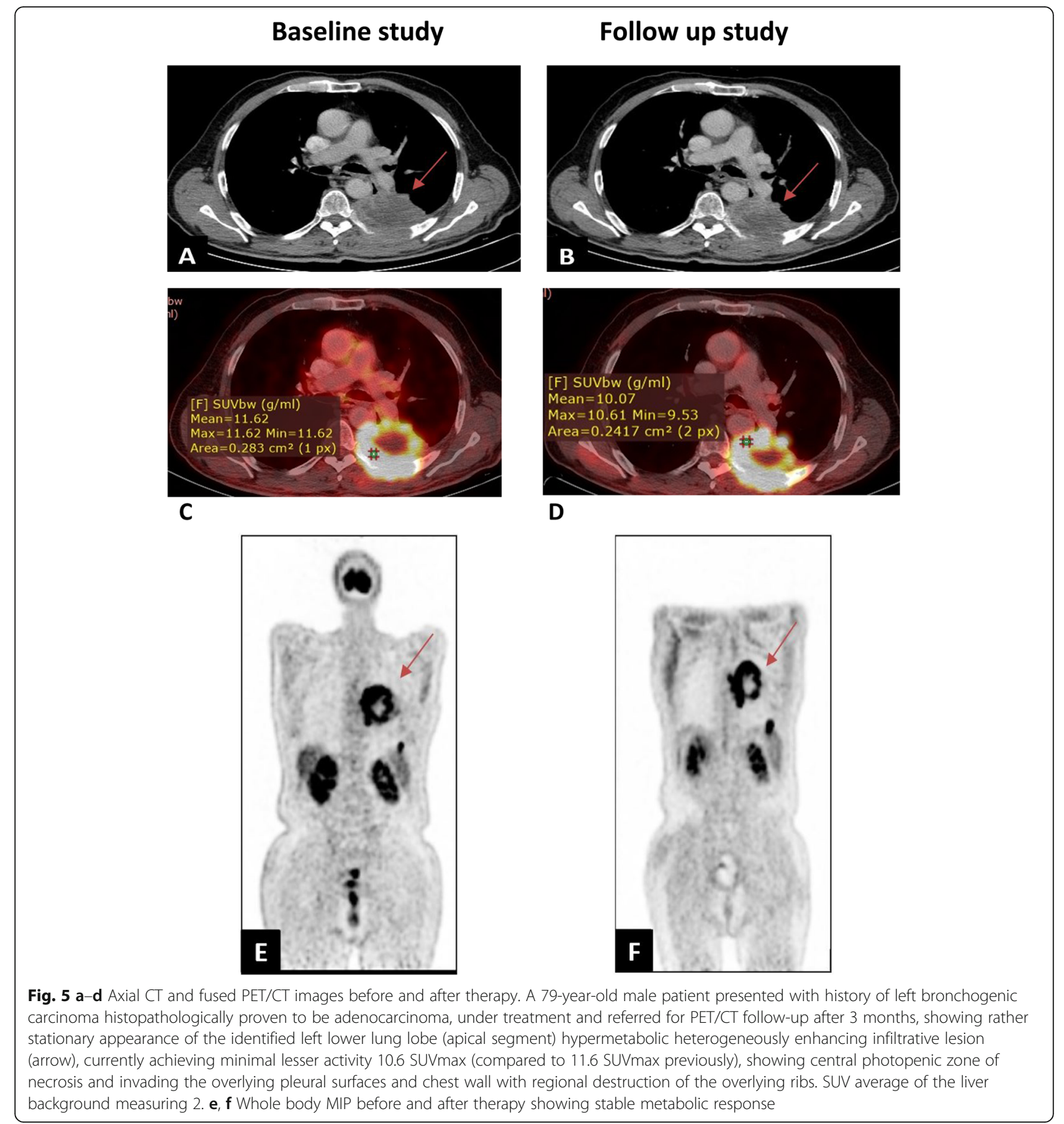

tumors, post-therapeutic changes, or tumors associated with distal atelectasis [33].

Additionally, William et al. and Cerfolio et al. found a mismatch between the CT RECIST and the histopathological results among the studied cases during follow-up after chemotherapy. They stated that CT RECIST may have a limited role in histopathologic response assessment and prediction of survival in patients with NSCLC. Also, Erasmus et al. concluded that RECIST tend to misclassify tumor progression in $30 \%$ of lesions in their study [24, 34, 35].

This is consistent with Mac Manus et al. who studied the value of FDG PET and enhanced CT in NSCLC patients before and after chemo-radiotherapy and found poor agreement between metabolic PET and morphologic CT response categories which were identical in only $40 \%$ of the studied cases [36]. 


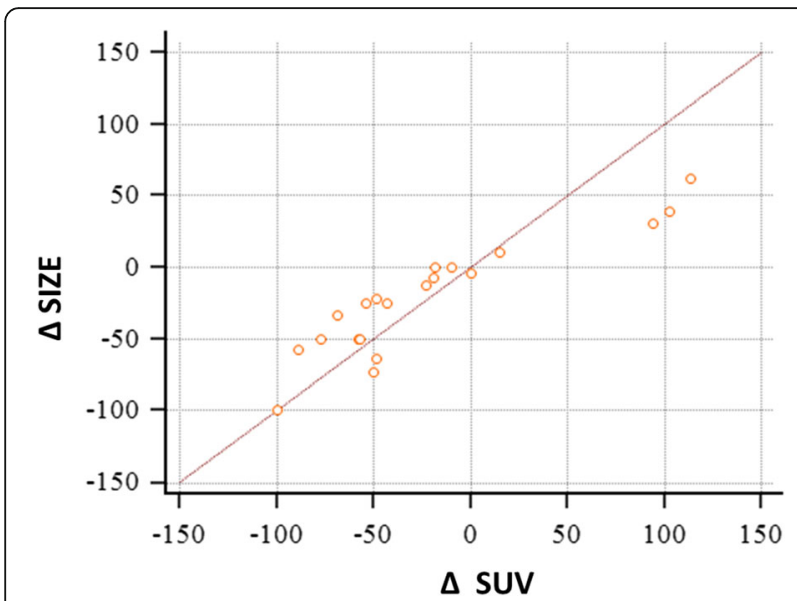

Fig. 6 Scatter diagram displaying the strong positive correlation between $\triangle S U V$ and $\triangle S I Z E$ in all patients

The limitations of the current study were that PET-CT is relatively more expensive than other scanning modalities, and this must be considered in the current financial climate; another limitation was the small sample size. Further studies with large patient numbers are needed to validate the results of our study.

\section{Conclusion}

In conclusion, SUVmax of the primary tumor changes from two serial 18F-FDG PET/CT studies, before and after therapy, allows prediction of the treatment response in patients with bronchogenic carcinoma, which leads to a significant change in therapeutic and management strategy by avoiding ineffective chemoradiotherapy and supporting the decision to continue the primary treatment in responding patients, and this hopefully will improve the patient's outcome and prognosis. In this study, a close correlation was found between SUVmax and response to therapy according to RECIST criteria. Although RECIST criteria is widely accepted and considered as a useful surrogate marker for quick assessment of tumor response to treatment, it has some limitations as changes in CT-measured tumor size are unreliable in predicting histopathologic response after therapy.

Finally, FDG-PET/CT proved itself as a useful, efficient, and reliable tool of investigation in initial staging and follow-up of lung cancer patients, and recently became a part of the international guidelines in the diagnosis and the follow-up of cases with bronchogenic carcinoma. Also, it has shown promise in monitoring response to biological agents such as epidermal growth factor receptor tyrosine kinase inhibitors.

\section{Abbreviations}

AUC: Area under the curve; CR: Complete response; CT: Computed tomography; 18 F-FDG: 18 F-Fluorodeoxeglucose; NPV: Negative predictive value; NSCLC: Non-small cell lung cancer; PD: Progressive disease; PERC IST: PET response criteria in solid tumors; PET: Positron emission tomography; PPV: Positive predictive value; PR: Partial response; RECIST: Response Evaluation Criteria in solid tumors; ROC: Receiver operator characteristic; SCLC: Small cell lung cancer; SD: Stable disease; SUV: Standardized uptake value; SUVmax: Maximum standardized uptake value; TNM: Tumor node metastasis

\section{Acknowledgements}

The authors thank all the study participants for their patience and support. This research was carried out at Tahra Radiology Center and Misr Radiology Center which are fully equipped with advanced PET/CT machines. The contributions of Prof. Dr. Essam ElSheikh, Prof. Dr. Hany Samy, and Dr. Yehia Omar Hussein to the practical part are gratefully acknowledged. Finally, statistical preparation was provided by Prof. Dr. Eman Darwish, to whom I would like to express my deepest gratitude for her generous advices, kind cooperation, and endless ideas for this work to be achieved.

\section{Authors' contributions}

All authors have read and approved the manuscript. A.S (Corresponding author): research study conception, participation in practical part, paper editing and revision, interpretation of data and statistical preparation, references collection, approval of the submitted version, agreed to be personally accountable for the author's own contributions. A.M: interpretation of data, revision of the work and paper editing, approval of the submitted version, agreed to be personally accountable for the author's own contributions. A.E.S: interpretation of data, revision of the work and paper editing, approval of the submitted version, agreed to be personally accountable for the author's own contributions. M.H: interpretation of data, revision of the work and paper editing, approval of the submitted version, agreed to be personally accountable for the author's own contributions.

Funding

This study had no funding from any resource.

\section{Availability of data and materials}

The datasets used and/or analyzed during the current study are available from the corresponding author on reasonable request.

\section{Declarations}

\section{Ethics approval and consent to participate}

This study was approved by the Research Ethics Committee of the Faculty of Medicine, Ain Shams University in Egypt on 05/09/2018; reference number of approval: FWA000017585. FMASU M D 279/ /2018.

A written informed consent was obtained from each patient.

\section{Consent for publication}

A written informed consent was obtained from all patients included in this study for their participation.

\section{Competing interests}

The authors declare that they have no competing interests.

Received: 7 December 2020 Accepted: 25 April 2021

Published online: 17 May 2021

\section{References}

1. Gadgeel SM, Ramalingam SS, Kalemkerian GP (2012) Treatment of lung cancer. Radiol Clin N Am 50(5):961-974. https://doi.org/10.1016/j.rcl.2012. 06.003

2. Sheikhbahaei S, Mena E, Yanamadala A, Reddy S, Solnes LB, Wachsmann J, Subramaniam RM (2017) The value of FDG PET/CT in treatment response assessment, follow-up, and surveillance of lung cancer. AJR 208(2):420-433. https://doi.org/10.2214/AJR.16.16532

3. Therasse P, Arbuck SG, Eisenhauer EA, Wanders J, Kaplan RS, Rubinstein L, Verweij J, van Glabbeke M, van Oosterom AT, Christian MC, Gwyther SG (2000) New guidelines to evaluate the response to treatment in solid tumors. European Organization for Research and Treatment of Cancer, National Cancer Institute of the United States, National Cancer Institute 
of Canada. J Natl Cancer Inst 92(3):205-216. https://doi.org/10.1093/jnci/ 92.3.205

4. Eisenhauer EA, Therasse P, Bogaerts J, Schwartz LH, Sargent D, Ford R, Dancey J, Arbuck S, Gwyther S, Mooney M, Rubinstein L, Shankar L, Dodd L, Kaplan R, Lacombe D, Verweij J (2009) New response evaluation criteria in solid tumors revised RECIST guidelines (version 1.1). Eur J Cancer 45(2):228247. https://doi.org/10.1016/j.ejca.2008.10.026

5. Nishino M, Jackman DM, Hatabu H, Yeap BY, Cioffredi LA, Yap JT, Jänne PA, Johnson BE, van den Abbeele AD (2010) New Response Evaluation Criteria in Solid Tumors (RECIST) guidelines for advanced non-small cell lung cancer: comparison with original RECIST and impact on assessment of tumor response to targeted therapy. AJR Am J Roentgenol 195(3):W221W228. https://doi.org/10.2214/AJR.09.3928

6. Coche E (2013) RECIST and beyond. JBR-BTR 96(3):167-171. https://doi.org/1 $0.5334 / j \mathrm{jbr}-\mathrm{btr} .242$

7. Coche $E$ (2016) Evaluation of lung tumor response to therapy: current and emerging techniques. Diagn Interv Imaging 97(10):1053-1065. https://doi. org/10.1016/j.diii.2016.09.001

8. Baheti AD, Rastogi A, Patra A et al (2019) Tumor response criteria in oncoimaging: RECIST criteria and beyond —Part 2. J Gastrointest Abdom Radiol 2(02):098-106

9. Dunlap NE, Yang W, Mclntosh A, Sheng K, Benedict SH, Read PW, Larner JM (2012) Computed tomography-based anatomic assessment overestimates local tumor recurrence in patients with mass like consolidation after stereotactic body radiotherapy for early-stage non-small cell lung cancer. Int J Radiat Oncol Biol Phys 84(5):1071-1077. https//doi.org/10.1016/j.jpobp.2012.01.088

10. Huang W, Fan M, Liu B, Fu Z, Zhou T, Zhang Z, Gong H, Li B (2014) Value of metabolic tumor volume on repeated 18F-FDG PET/CT for early prediction of survival in locally advanced non-small cell lung cancer treated with concurrent chemoradiotherapy. J Nucl Med 55(10):1584-1590. https://doi. org/10.2967/jnumed.114.142919

11. Khiewvan B, Pouya Z, Houshmand S et al (2016) The role of PET/CT as a prognosticator and outcome predictor in lung cancer. Expert Rev Respir Med 15(34):31

12. Lee JW, Lee SM, Yun M, Cho A (2016) Prognostic value of volumetric parameters on staging and posttreatment FDG PET/CT in patients with stage IV non-small cell lung cancer. Clin Nucl Med 41(5):347-353. https:// doi.org/10.1097/RLU.0000000000001126

13. Nishino M, Hatabu H, Johnson BE, McLoud TC (2014) State of the art: response assessment in lung cancer in the era of genomic medicine. Radiology 271(1):6-27. https://doi.org/10.1148/radiol.14122524

14. Larici AR, del Ciello A, Maggi F, Santoro SI, Meduri B, Valentini V, Giordano A, Bonomo L (2011) Lung abnormalities at multimodality imaging after radiation therapy for non-small cell lung cancer. RadioGraphics 31(3):771789. https://doi.org/10.1148/rg.313105096

15. Detterbeck FC, Boffa DJ, Tanoue LT (2009) The new lung cancer staging system. Chest 136:260-271

16. Akl YM, Emam RH, Sabry IM, Ali AA (2013) Clinico-pathological profile of bronchogenic carcinoma cases presented to Chest Department, Cairo University in the last 10 years. Egypt J Chest Dis Tuberc 62(4):705-712 https://doi.org/10.1016/.jejcdt.2013.09.019

17. Chao F, Zhang H (2012) PET/CT in the staging of the non-small cell lung cancer. J Biomed Biotechnol 2012:8

18. Steinert HC (2011) PET and PET-CT of lung cancer. Methods Mol Biol 727: 33-51. https://doi.org/10.1007/978-1-61779-062-1_3

19. Aydin F, Dertsiz L, Budak E, Yildiz A, Ozbilim G, Gungor F (2013) Measurements of tumor size using CT and PET compared to histopathological size in non-small cell lung cancer. Diagn Interv Radiol 19(4):271-278. https://doi.org/10.5152/dir.2013.053

20. Hochhegger $B$, Alves $G$, Irion $K$ et al (2015) PET/CT imaging in lung cancer: indications and findings. J Bras Pneumol 41(3):264-274. https://doi.org/10.1 590/S1806-37132015000004479

21. Bahce I, Vos CG, Dickhoff C, Hartemink K, Dahele M, Smit EF, Boellaard R, Hoekstra OS, Thunnissen E (2014) Metabolic activity measured by FDG PET predicts pathological response in locally advanced superior sulcus NSCLC. Lung Cancer 85(2):205-212. https://doi.org/10.1016/.lungcan.2014.04.010

22. Yamamoto $Y$, Nishiyama $Y$, Monden $T$, Sasakawa $Y$, Ohkawa M, Gotoh M, Kameyama K, Haba R (2006) Correlation of FDG-PET findings with histopathology in the assessment of response to induction chemoradiotherapy in non-small cell lung cancer. Eur J Nucl Med Mol Imaging 33(2):140-147. https://doi.org/10.1007/s00259-005-1878-9
23. Huang W, Zhou T, Ma L, Sun H, Gong H, Wang J, Yu J, Li B (2011) Standard uptake value and metabolic tumor volume of (1)(8)F-FDG PET/CT predict short-term outcome early in the course of chemoradiotherapy in advanced non-small cell lung cancer. Eur J Nucl Med Mol Imaging 38(9):1628-1635. https://doi.org/10.1007/s00259-011-1838-5

24. Cerfolio RJ, Bryant AS, Winokur TS et al (2004) Repeat FDG-PET after neoadjuvant therapy is a predictor of pathologic response in patients with non-small cell lung cancer. Ann Thorac Surg 78:1903-1909 discussion, 1909

25. Lee DH, Kim SK, Lee HY, Lee SY, Park SH, Kim HY, Kang KW, Han JY, Kim HT, Lee JS (2009) Early prediction of response to first-line therapy using integrated 18FFDG PET/CT for patients with advanced/metastatic non-small cell lung cancer. J Thorac Oncol 4(7):816-821. https://doi.org/10.1097/JTO.0b013e3181a99fde

26. Eschmann SM, Friedel G, Paulsen F, Reimold M, Hehr T, Budach W, Langen HJ, Bares R (2007) 18 F-FDG PET for assessment of therapy response and preoperative re-evaluation after neoadjuvant radio-chemotherapy in stage III non-small cell lung cancer. Eur J Nucl Med Mol Imaging 34(4):463-471. https://doi.org/10.1007/s00259-006-0273-5

27. Rastogi A, Baheti AD, Patra A et al (2019) Tumor response criteria in oncoimaging: RECIST criteria and beyond-Part 1. J Gastrointest Abdom Radiol 2(02):098-106

28. Fattah AS, Refaat MM, Shahin MA (2017) Role of PET/CT in staging and assessment the therapy response of lung cancer. ResearchGate https:// www.researchgate.net/publication/316254621_ROLE_OF_PETCT_IN_STA GING_AND_ASSESSMENT_THE_THERAPY_RESPONSE_OF_LUNG_CANCER

29. Cappabianca S, Porto A, Petrillo M, Greco B, Reginelli A, Ronza F, Setola F, Rossi G, di Matteo A, Muto R, de Rimini ML, Piccolo S, Catalano M, Muto P, de Rosa N, Barra E, de Rosa I, Antinolfi F, Antinolfi G, Caputi M, Brunese L, Grassi R, Rotondo A (2011) Preliminary study on the correlation between grading and histology of solitary pulmonary nodules and contrast enhancement and [18 F] fluorodeoxyglucose standardised uptake value after evaluation by dynamic multiphase CT and PET/CT. J Clin Pathol 64(2): 114-119. https://doi.org/10.1136/jcp.2010.076562

30. Lu P, Yu L, Li Y, Sun Y (2010) A correlation study between maximum standardized uptake values and pathology and clinical staging in non-small cell lung cancer. Nucl Med Commun 31(7):646-651. https://doi.org/10.1097/ MNM.0b013e328339bddb

31. Khalaf M, Abdel-Nabi H, Baker J, Shao Y, Lamonica D, Gona J (2008) Relation between nodule size and 18F-FDG-PET SUV for malignant and benign pulmonary nodules. J Hematol Oncol 1(1):13. https://doi.org/10.1186/17568722-1-13

32. Weber WA, Petersen V, Schmidt B, Tyndale-Hines L, Link T, Peschel C, Schwaiger M (2003) Positron emission tomography in non-small-cell lung cancer: prediction of response to chemotherapy by quantitative assessment of glucose use. J Clin Oncol 21(14):2651-2657. https://doi.org/10.1200/JCO.2 003.12 .004

33. Hicks RJ (2009) Role of 18F-FDG PET in assessment of response in non-small cell lung cancer. J Nucl Med 50(suppl 1):31S-42S. https://doi.org/10.2967/ jnumed.108.057216

34. William W, Pataer A, Kalhor N et al (2013) Computed tomography RECIST assessment of histopathological response and prediction of survival in patients with resectable non-small cell lung cancer after neoadjuvant chemotherapy. J Thorac Oncol 8(2):222-228. https://doi.org/10.1097/JTO. ob013e3182774108

35. Erasmus JJ, Gladish GW, Broemeling L et al (2003) Interobserver and intraobserver variability in measurement of non-small-cell carcinoma lung lesions: implications for assessment of tumor response. J Clin Oncol 21: 2574-2582

36. Mac Manus MP, Hicks RJ, Matthews JP, McKenzie A, Rischin D, Salminen EK, Ball DL (2003) Positron emission tomography is superior to computed tomography scanning for response-assessment after radical radiotherapy or chemoradiotherapy in patients with non-small-cell lung cancer. J Clin Oncol 21(7):1285-1292. https://doi.org/10.1200/JCO.2003.07.054

\section{Publisher's Note}

Springer Nature remains neutral with regard to jurisdictional claims in published maps and institutional affiliations. 